\title{
Mechanisms of radiative and nonradiative recombination in ZnSe:Cr and ZnSe:Fe
}

\author{
M. Godlewski ${ }^{1,2}$, M. Surma $^{1}$, V.Yu. Ivanov ${ }^{1}$, and T.P. Surkova ${ }^{3}$ \\ ${ }^{1}$ Institute of Physics of Polish Academy of Sciences, 32/46 Al. Lotnikow Str., Warsaw 02-668, Poland \\ E-mail: godlew@ifpan.edu.pl \\ ${ }^{2}$ Department of Mathematics and Natural Sciences College of Science, Cardinal S. Wyszyński \\ University, Warsaz, Poland \\ ${ }^{3}$ Institute of Metal Physics of Russian Academy of Sciences, Ural Division, Ekaterinburg, Russia
}

Received May 25, 2004

\begin{abstract}
Possible applications of $\mathrm{ZnSe}: \mathrm{Cr}$ in optoelectronics are discussed. It is shown that $2+$ to $1+$ photo-ionization of chromium results in efficient pumping of $\mathrm{Cr}^{2+}$ intrashell emission and in energy up-conversion from green to blue. A distinct difference in efficiency of the energy up-conversion is observed between chromium and iron doped $\mathrm{ZnSe}$ samples. This difference we relate to a very efficient Auger mechanism of photoluminescence quenching in Fe-doped samples. We further demonstrate an anticorrelation of intensity of mid-infrared $\mathrm{Cr}$ emission and up-converted blue emission of $\mathrm{ZnSe}$.
\end{abstract}

PACS: 42.55.Px, 71.55.Gs, 78.55.Et

\section{Introduction}

Despite concentrated efforts there are difficulties in achieving efficient short-wavelength emission from semiconductor-based light emitting devices. As a consequence, several alternative approaches have been tested, in which green, blue, or violet color emission was obtained due to energy up-conversion, i.e., under optical pumping with photons of a lower energy (longer wavelength).

$\mathrm{ZnSe}$ is an attractive candidate for the short wavelength energy up-conversion material, since the «edge» (i.e., close to the band gap energy) photoluminescence (PL) of ZnSe is observed in blue-violet spectral region. Unfortunately, as we have found [1], two-photon excitation is an inefficient process in the case of undoped ZnSe. Quantum efficiency of the process is very low, of about $10^{-6}$, i.e., is far too low for any practical application. A very different situation was observed in the case of chromium-doped samples $[1,2]$. Relatively efficient blue up-converted emission was observed under green color optical pumping.

In the following Section we will discuss the mechanism of energy up-conversion in $\mathrm{Cr}$ doped $\mathrm{ZnSe}$, and then we will discuss the possibility of tuning of pump- ing energy by doping ZnSe with iron or by using alloys of wide-band-gap II-VI semiconductors. Finally, we will discuss the properties of infrared emission in $\mathrm{ZnSe}: \mathrm{Cr}$.

\section{2. $\mathrm{ZnSe}: \mathrm{Cr}-$ mechanism of energy up-conversion}

In Figs. 1 and 2 we show photo-ionization (Fig. 1) and photo-neutralization (quenching, Fig. 2) spectra measured for $\mathrm{Cr}^{1+}$ and $\mathrm{Fe}^{3+}$ electron spin resonance (ESR) signals in $\mathrm{ZnSe}$. Two complementary $\mathrm{Cr}$ ionization transitions were identified in this ESR study as:

$$
\mathrm{Cr}^{2+}+\text { photon } \rightarrow \mathrm{Cr}^{1+}+\text { hole in the valence band }
$$

(band II in Fig. 1),

$\mathrm{Cr}^{1+}+$ photon $\rightarrow \mathrm{Cr}^{2+}+$ electron in the conduction band

(direct photo-ionization in Fig. 2),

with $\mathrm{Cr}^{2+}$ being the ground charge state of chromium in $\mathrm{ZnSe}$.

$\mathrm{Cr}^{1+}$ signal could also be excited by illumination ionizing acceptor centres of $\mathrm{ZnSe}$ (band III in Fig. 1) and, for $\mathrm{ZnSe}: \mathrm{Fe}, \mathrm{Cr}$, by the 2+ to 3+ photo-ionization transition of $\mathrm{Fe}$ ions (band $\mathrm{I}$ in Fig. 1,b,c), i.e., by 


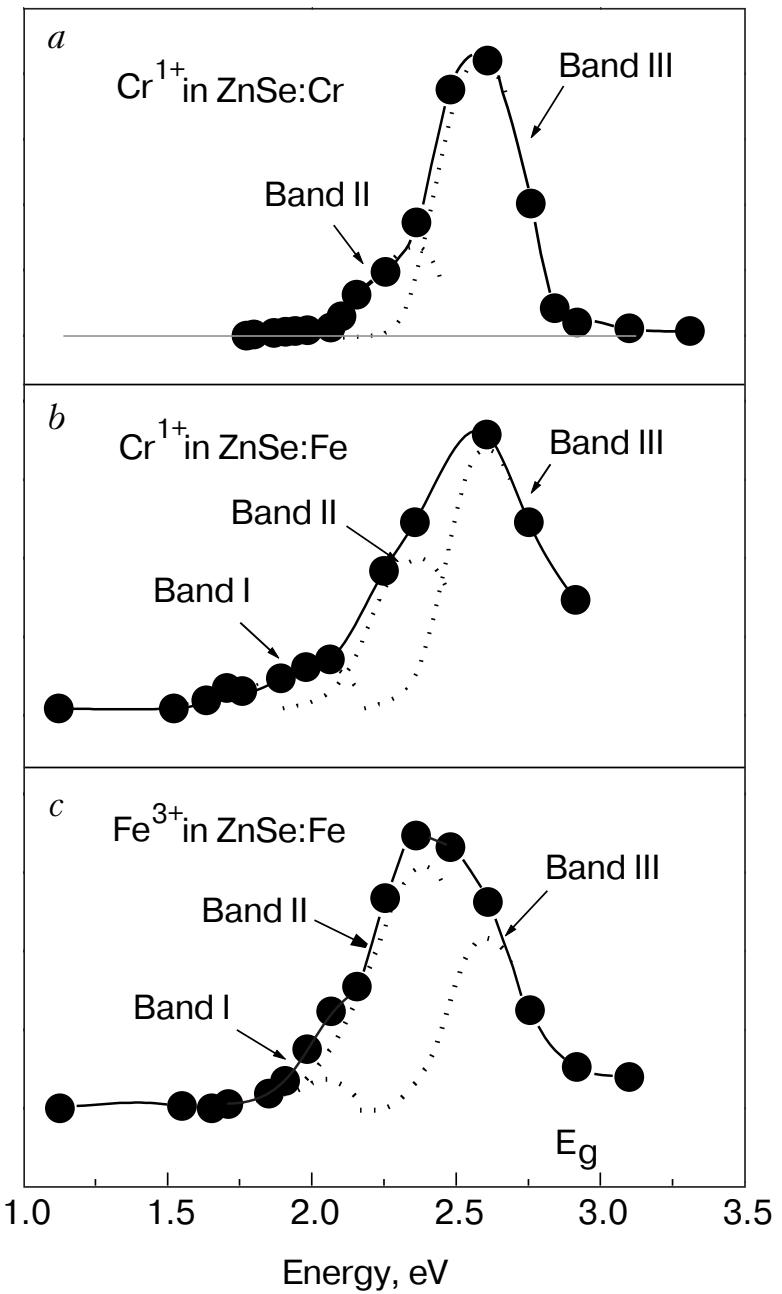

Fig. 1. Low temperature photo-excitation spectra of $\mathrm{Cr}^{1+}$ in $\mathrm{ZnSe}: \mathrm{Cr}(a), \mathrm{ZnSe}: \mathrm{Fe}(b)$ and $\mathrm{Fe}^{3+}$ in $\mathrm{ZnSe}: \mathrm{Fe}(c)$.

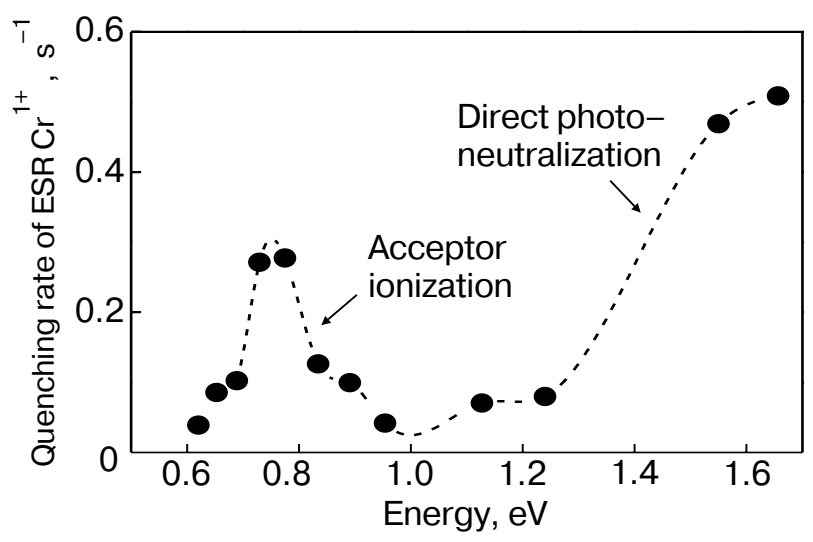

Fig. 2. Low temperature photo-quenching spectrum of the $\mathrm{Cr}^{1+}$ ESR signal in $\mathrm{ZnSe}: \mathrm{Cr}$, measured as the spectral dependence of the decay time of the ESR signal under illumination with photons of a given energy. The $\mathrm{Cr}^{1+}$ ESR signal was first photo-excited with green color illumination. two transitions generating free electrons in the conduction band of $\mathrm{ZnSe}$. These electrons can be retrapped by chromium resulting in $2+$ to $1+$ recharging.

Such identity of the ESR excitation and quenching bands was proved in our previous ESR experiments [3-7]. We found that $2+/ 1+$ energy level of $\mathrm{Cr}$ lies at about $2 \mathrm{eV}$ above the valence band edge of $\mathrm{ZnSe}$ [4]. The complementary $1+\rightarrow 2+$ photo-neutralization transition was also identified, as shown in Fig. 2.

Our previous ESR studies of Cr-doped ZnSe [4] and $\mathrm{ZnS}$ [5] and iron-doped $\mathrm{ZnSe}$ [6] and $\mathrm{ZnS}$ [7] indicated high efficiency of these ionization transitions in wide-band gap II-VI semiconductors. Moreover, we observed that two-step ionization transitions of $\mathrm{Cr}$ and Fe ions result in a population of both shallow donors and shallow and deep acceptors [8].

In the $2+\rightarrow 1+$ chromium photo-ionization transition free holes are photo-generated in the valence band (VB). In the following $1+\rightarrow 2+$ photo-neutralization transition free electrons are created in the conduction band $(\mathrm{CB})$. These free carriers, if not retrapped by chromium (iron), can then participate in the PL recombination transitions, resulting, e.g., in the appearance of the energy up-converted emission. Not surprisingly, we found that the photoconductivity and photo-ionization (due to $2+$ to $1+\mathrm{Cr}$ transition) spectra of $\mathrm{ZnSe}$ : Cr both correlate with the excitation spectrum of up-converted emission [1]. Thus, energy up-conversion is explained by efficient photo-generation of free electrons and free holes in two complementary photo-ionization transitions via deep chromium-related center.

\section{ZnSe:Fe - optimization of optical pumping energy}

The blue up-converted emission of $\mathrm{ZnSe}: \mathrm{Cr}$ is excited by the $2+\rightarrow 1+$ photo-ionization transition of chromium, which occurs for photon energies larger than about $2 \mathrm{eV}$, with maximum at about $2.4 \mathrm{eV}$ [1]. Considering possible practical devices, the use of efficient red color GaAs-based laser diodes for optical pumping is preferential. Only in this case can compact devices be constructed, as required for memory storage applications, for example.

Regarding energy up-conversion efficiency, light powers of several $\mathrm{mW}$ are required for energy storage applications. Considering that $1 \mathrm{~W}$ optical pumping is available, the light conversion efficiency should be not less than few times $10^{-3}$.

For ZnSe:Cr up-conversion efficiency was about $5 \cdot 10^{-3}$ at liquid helium temperature, but only for green color optical pumping. For red pumping up-con- 


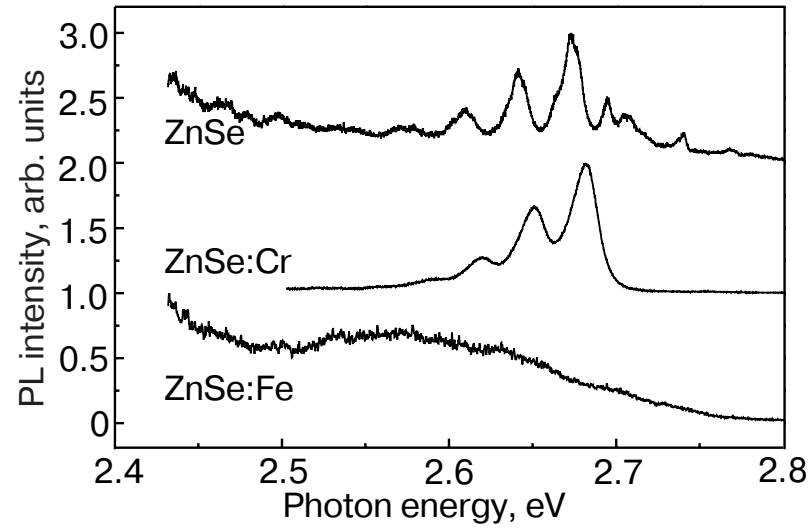

Fig. 3. «Edge» part of the $\mathrm{PL}$ in $\mathrm{ZnSe}, \mathrm{ZnSe}: \mathrm{Cr}$ and $\mathrm{ZnSe}: F e$ measured at liquid helium temperature under the green color excitation (for $\mathrm{ZnSe:Cr}$ and $\mathrm{ZnSe}: \mathrm{Fe}$ ) or above-band-gap excitation (ZnSe undoped) (after V.Yu. Ivanov et al., Acta Phys. Pol. A103, 695 (2003)).

version efficiency was about 10 times lower, i.e., too low for practical applications.

A photo-ESR investigation (Fig. 1) indicated that red color pumping is optimized for $2+$ to $3+$ photo-ionization of iron in ZnSe. We thus turned our attention to this system. In Fig. 3 we show the energy up-converted PL spectra in chromium and iron doped $\mathrm{ZnSe}$, as compared to the «edge» PL of an undoped $\mathrm{ZnSe}$. The origin of the observed PL emissions in ZnSe and $\mathrm{ZnSe}: \mathrm{Cr}$ is discussed elsewhere [1]. Weak and broad blue color energy up-converted emission is observed in $\mathrm{ZnSe}: \mathrm{Fe}$. Its efficiency is far too low to be of any practical interest. The quantum efficiency of energy up-conversion in $\mathrm{ZnSe}: \mathrm{Fe}$ we estimated to be two or-

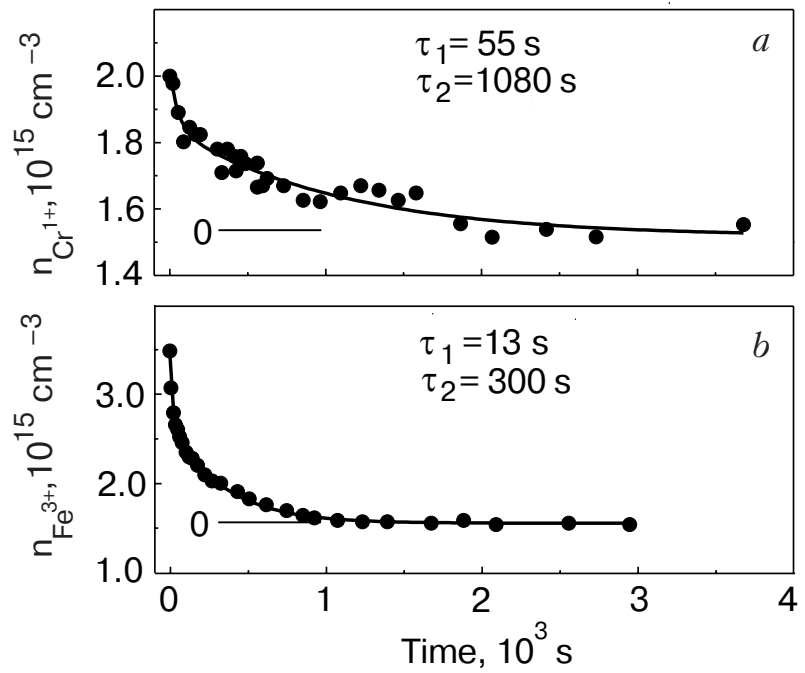

Fig. 4. Decay of $\mathrm{Cr}^{1+}(a)$ and $\mathrm{Fe}^{3+}(b)$ ESR signals observed at $40 \mathrm{~K}$ after turning off the photo-excitation. Two-exponential decay is observed, with the characteristic times given in the figure.

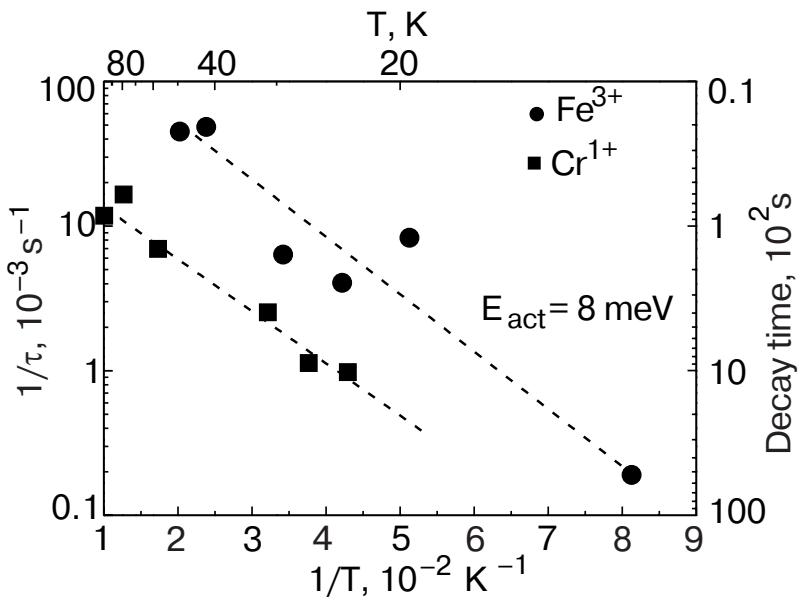

Fig. 5. Temperature dependence of decay of the $\mathrm{Cr}^{1+}$ and $\mathrm{Fe}^{3+}$ ESR signals observed after turning off the photo-excitation. Two decay components (see Fig. 4) are characterized by the same temperature dependence with an activation energy of $8 \mathrm{meV}$.

ders of magnitude smaller than the one for chromium doped ZnSe.

Our present investigations indicate that two mechanisms are responsible for the low efficiency of the energy up-conversion process in $\mathrm{ZnSe}: \mathrm{Fe}$. First, the photo-ESR investigations indicate that the photo-excited $\mathrm{Fe}^{3+}$ charge state of iron decays fast even at low temperatures. The mid-gap level of $\mathrm{Fe}$ is not metastably occupied, as is often observed in the case of chromium-doped $\mathrm{ZnSe}$. This relates to high efficiency of carrier trapping processes in iron-doped $\mathrm{ZnS}$ and $\mathrm{ZnSe}$ [9-11]. The photogenerated $\mathrm{Fe}^{3+}$ state has too large a cross section for retrapping of free electrons from the conduction band to be metastably occupied after the photogeneration. The latter we concluded from detail studies of kinetics of quenching of photo-excited ESR signals. Examples of relevant ESR results are shown in Figs. 4 and 5 for $\mathrm{ZnSe}: \mathrm{Fe}, \mathrm{Cr}$. Both $\mathrm{Cr}^{1+}$ and $\mathrm{Fe}^{3+}$ ESR signals decay fast after turning off the excitation to some metastable population. Decay was faster for Fe ions. In both cases the decay was characterized by the same activation energy equal to ionization energy of shallow donors in $\mathrm{ZnSe}$. The latter was surprising in case of $\mathrm{Cr}^{1+}$ centers, for which rise of the signal was observed in $\mathrm{ZnSe}: \mathrm{Cr}$, but decay in $\mathrm{ZnSe}: \mathrm{Fe}$, Cr once electrons were thermally ionized. The reason for this difference we will discuss later on.

We solved simple kinetics equations to explain evolution of ESR signals under photo-excitation and after turning off the light:

$$
\begin{aligned}
& \frac{d n_{\mathrm{Cr}}}{d t}=I \sigma_{O V}\left(N_{\mathrm{Cr}}-n_{\mathrm{Cr}}\right)-I \sigma_{O C} n_{\mathrm{Cr}}+ \\
& +n\left(N_{\mathrm{Cr}}-n_{\mathrm{Cr}}\right) c_{\mathrm{Cr}}^{e}-p n_{\mathrm{Cr}} c_{\mathrm{Cr}}^{h},
\end{aligned}
$$




$$
\begin{gathered}
\frac{d n}{d t}=I \sigma_{O C} n_{\mathrm{Cr}}-n\left(N_{D}-n_{D}\right) c_{D}^{e}-n\left(N_{\mathrm{Cr}}-n_{\mathrm{Cr}}\right) c_{\mathrm{Cr}}^{e}, \\
\frac{d p}{d t}=I \sigma_{O V}\left(N_{\mathrm{Cr}}-n_{\mathrm{Cr}}\right)-p\left(N_{A}-n_{A}\right) c_{A}^{h}-p n_{\mathrm{Cr}} c_{\mathrm{Cr}}^{h}, \\
\frac{d n_{A}}{d t}=p\left(N_{A}-n_{A}\right) c_{D}^{e}-\beta_{A D} n_{A} n_{D}-\beta_{A \mathrm{Cr}} n_{A} n_{\mathrm{Cr}}, \\
\frac{d n_{D}}{d t}=n\left(N_{D}-n_{D}\right) c_{D}^{e}-\beta_{A D} n_{A} n_{D},
\end{gathered}
$$

where: $n_{\mathrm{Cr}}$ and $N_{\mathrm{Cr}}$ are the concentration of the chromium in the $1+$ charge state and the total concentration of $\mathrm{Cr}$ in the sample, respectively; $N_{A}$ and $N_{D}$ are the total concentrations of acceptors (A) and donors (D) in the sample, $n_{A}$ and $n_{D}$ are the concentrations of populated (neutral) acceptors and donors. $n, p$ are the concentrations of free electrons in the conduction band and free holes in the valence band; $\sigma_{O C}$ and $\sigma_{O V}$ are the optical ionization rates for the two complementary ionization transitions of $\mathrm{Cr}$. By $c_{D}^{e}, c_{A}^{h}$, $c_{\mathrm{Cr}}^{e}$ and $c_{\mathrm{Cr}}^{h}$ we denote the capture rates of electrons $(e)$ by ionized donors, holes $(h)$ by ionized acceptors, and electrons by chromium $\mathrm{Cr}^{2+}$ and holes by $\mathrm{Cr}^{1+}$. By $\beta_{D A P}$ we describe an average rate of the DA pairs (DAP) recombination, by $\beta_{A \mathrm{Cr}} \mathrm{Cr}^{1+}-$ acceptor tunneling and $I$ stands for the light intensity of the photo-excitation. In equations given above we omitted terms related to Auger processes, which will be discussed later on.

Detailed analysis of the observed efficiency of photo-excitation for bands II and III in Fig. 1 and rates of signal rise and decay indicated that:

$$
\frac{c_{\mathrm{Cr}}^{e}}{c_{D}^{e}}>25 \frac{N_{D}}{N_{A}} \frac{\sigma_{O V}^{\mathrm{Cr}}}{\sigma_{O C}^{A}} .
$$

Assuming that our samples are compensated, i.e. that $N_{D} \approx N_{A}$, and that:

$$
\frac{\sigma_{O V}^{C r}}{\sigma_{O C}^{A}}>\frac{1}{25}
$$

as we concluded from the separate study, and also that $\sigma_{O V}^{\mathrm{Cr}}$ is of the same order as $\sigma_{O C}^{\mathrm{Cr}}$, we derive that:

$$
\frac{\sigma_{O V}^{\mathrm{Cr}}}{\sigma_{O C}^{A}} \geq \frac{1}{10}
$$

and, finally, that $c_{\mathrm{Cr}}^{e} \geq 2 c_{D}^{e}$. This estimation indicates that $\mathrm{Cr}$ centers efficiently retrap photogenerated free electrons from CB. Thus, we must select appropriate relative concentrations of donor and acceptor centers versus chromium concentration to avoid PL deactivation and to achieve efficient energy up-conversion.
For $\mathrm{ZnSe}: \mathrm{Fe}, \mathrm{Cr}$ carrier retrapping is far more efficient. Based on the photo-ESR data, from the observed efficiency of band I (Fig. 1,c) processes, we estimate that:

$$
\frac{c_{\mathrm{Fe}}^{e}}{c_{\mathrm{Cr}}^{e}}=\frac{N_{\mathrm{Cr}}}{n_{\mathrm{Cr}}} \frac{N_{\mathrm{Fe}}}{n_{\mathrm{Fe}}} \frac{\sigma_{\mathrm{OC}}^{\mathrm{Fe}}}{\sigma_{\mathrm{OC}}^{\mathrm{Cr}}}
$$

so:

$$
\frac{c_{\mathrm{Fe}}^{e}}{c_{\mathrm{Cr}}^{e}}>1
$$

Moreover:

$$
\frac{c_{\mathrm{Fe}}^{h}}{c_{A}^{h}}>\frac{c_{\mathrm{Cr}}^{e}}{c_{D}^{e}} \frac{N_{\mathrm{Cr}}}{n_{\mathrm{Cr}}} \frac{\sigma_{O C}^{\mathrm{Fe}}}{\sigma_{O C}^{(\mathrm{Ir})}}>1 .
$$

These estimations indicate that Fe deactivates (by efficient retrapping of free carriers of both types) all competing recombination transitions in $\mathrm{ZnSe}$, even in the case of a relatively low Fe concentration. This in fact we observed experimentally, as is shown in Figs. 3 and 6.

The second mechanism responsible for a low efficiency of energy up-conversion in $\mathrm{ZnSe}$ :Fe relates to very efficient Auger type nonradiative recombination processes observed in Fe doped wide-band-gap II-VI compounds (see [2] and references therein). In the $\mathrm{Au}^{-}$ ger process an excited donor acceptor pair decays nonradiatively by energy transfer to a nearby iron center, which is ionized. DAP PL is thus deactivated.

In the case of $\mathrm{Fe}$ doped $\mathrm{ZnS}$ and $\mathrm{ZnSe}$ this process turned out to be efficient, which results in efficient quenching of energy up-converted blue color DAP emission and in shortening of the DAP PL decay time, as we observed for $\mathrm{ZnSe}: \mathrm{Fe}$ [2]. «Edge» DAP emission

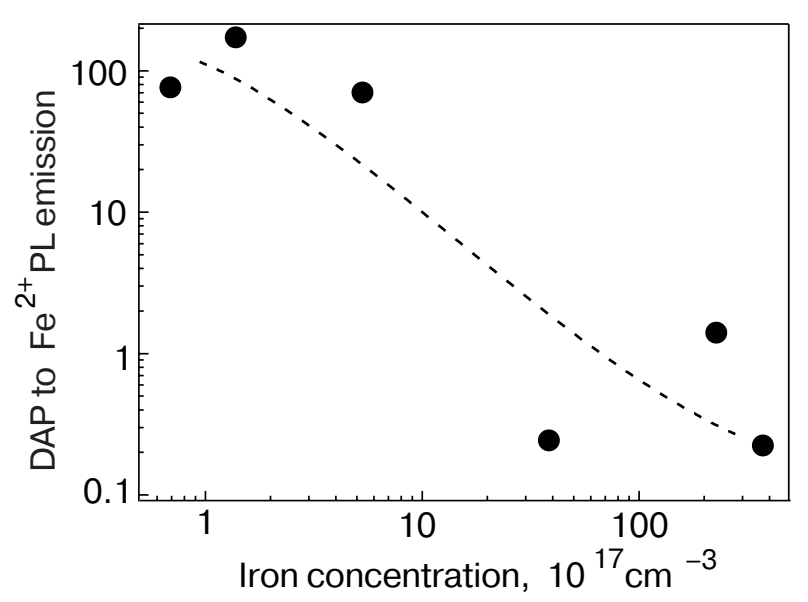

Fig. 6. Concentration dependence of deactivation of donor-acceptor pair emission in ZnSe:Fe. 
in $\mathrm{ZnSe}$ decays much faster than the relevant DAP emissions in undoped $\mathrm{ZnSe}$ and in $\mathrm{ZnSe}: \mathrm{Cr}$. This is evidence of very efficient and competing channel of nonradiative recombination in $\mathrm{ZnSe}: \mathrm{Fe}$.

Auger-type energy transfer must also take place in chromium doped $\mathrm{ZnSe}$, since also in this case there is an energy overlap between DAP emission and $\mathrm{Cr}$ ionization transitions. Such overlaps are required for efficient DAP-to-Cr energy transfer. We performed optically detected magnetic resonance (ODMR) investigations to evaluate Auger recombination rate in ZnSe:Cr [12]. The ODMR study indicated fairly low rate of DAP-to-Cr energy transfer process. This transfer process is rather inefficient in quenching DAP transitions of $\mathrm{ZnSe}: \mathrm{Cr}$.

\section{Solid alloys of II-VI:Cr - optimization of optical pumping energy}

Pumping energy of up-conversion process can be tuned by introducing chromium into solid alloys of $\mathrm{ZnCdSe}$ and $\mathrm{ZnSSe}$. Also in these alloys chromium introduces excited charge state within the band gap, so the alloys turned out to be suitable materials for energy up-conversion. The relevant results are shown in Fig. 7 , in which we show the CB and VB band shifts estimated by us versus the energy level positions of

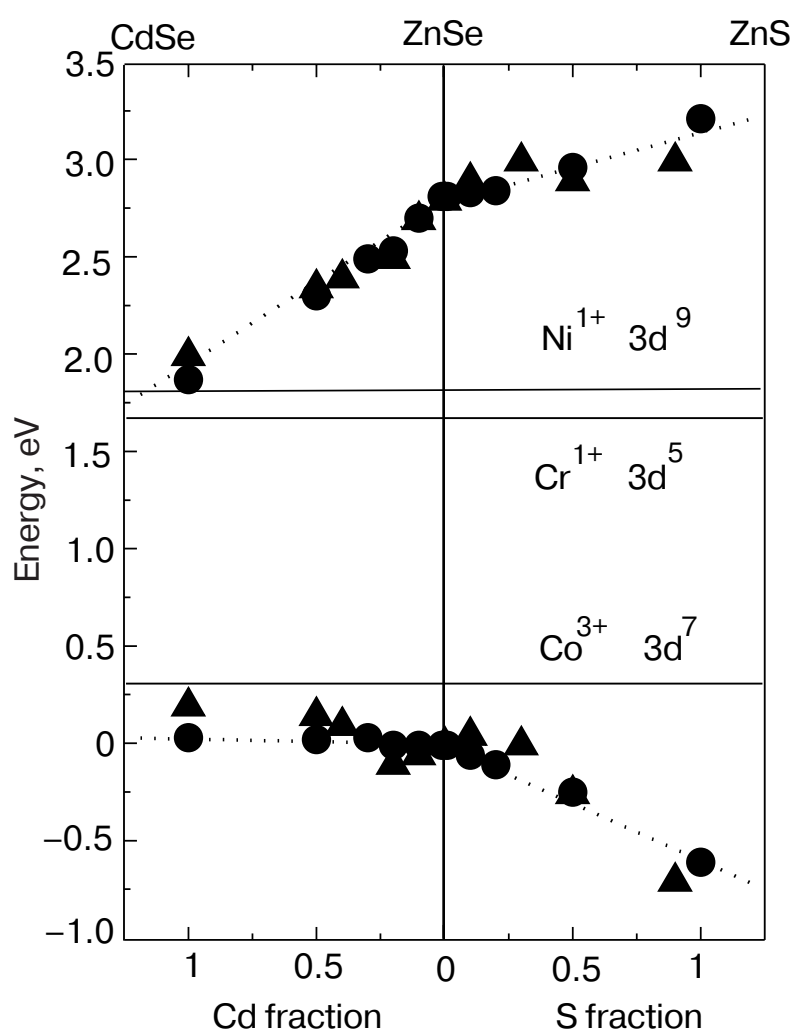

Fig. 7. Band shifts in solid alloys of ZnCdSe and ZnSSe versus energy level positions of three transition-metal-related charge states - nickel, cobalt, and chromium. three transition-metal-related charge state levels (nickel, cobalt and chromium) in $\mathrm{ZnCdSe}$ and $\mathrm{ZnSSe}$ (after Ref. [13]). Whereas for alloys with common anion $\mathrm{Cr}$ ionization energy changes only a little, it can be changed considerably in alloys of $\mathrm{ZnSSe}$ (increases) or $\mathrm{ZnCdS}$ (decreases; not shown in Fig. 7). These are important observations, since energy up-conversion in $\mathrm{Cr}$ doped $\mathrm{ZnCdS}$ and $\mathrm{ZnSSe}$ turned out to be equally efficient as in the case of $\mathrm{ZnSe:Cr}$.

\section{Temperature dependence of up-conversion process}

There is an additional important consequence of using chromium-doped II-VI alloys. Not only excitation (also energy of up-converted emission) can be tuned, but also temperature dependence of the process can be varied. This is important, since in the case of $\mathrm{ZnSe}$ quantum efficiency of the up-conversion process is reduced to about $1 \%$ at room temperature.

The photo-ESR investigations indicate that a relatively high quantum efficiency of the energy up-conversion process in $\mathrm{ZnSe}: \mathrm{Cr}$ (few times $10^{-3}$ ) is related to a metastable population of the $\mathrm{Cr}^{1+}$ state, observed at low temperatures. Two-color experiments were performed (Fig. 8) to confirm role of metastability of the photo-excited $\mathrm{Cr}^{1+}$ state. In the two-color experiments, two light sources were simultaneously applied to excite or quench the energy up-converted emission. First photon, with energy larger than the ZnSe band gap energy, or from the energy range of the $\mathrm{Cr} 2+$ to $1+$ ionization transition, excites the blue DAP emission. The second photon, selected from the range of ionization transitions of $\mathrm{ZnSe}$ acceptors. These ionization transitions were first identified from photo-ESR study shown in Fig. 2. The second illumination was

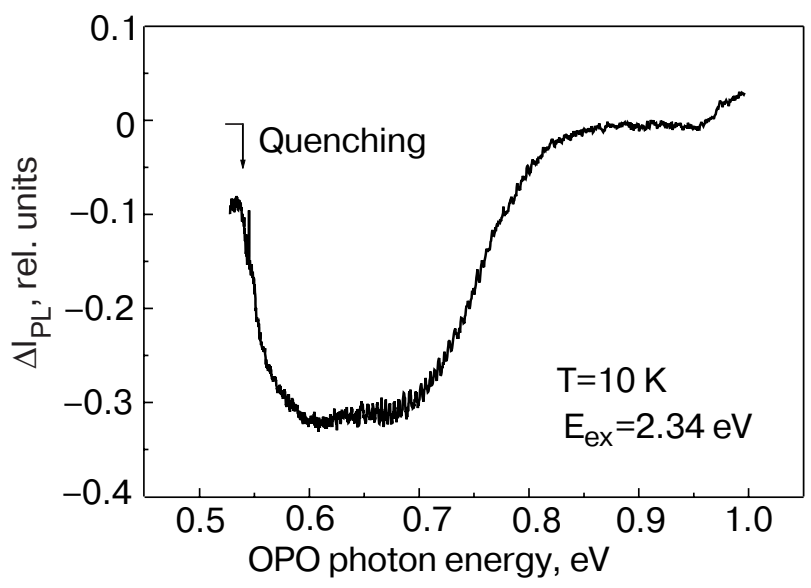

Fig. 8. Photo-quenching of the energy up-conversion process in $\mathrm{ZnSe}: \mathrm{Cr}$, observed in the two-color experiment with the OPO system and green color excitation. 
obtained from either the OPO system or the free-electron laser (FEL) mid-infrared system. We studied the influence of this second illumination on intensity of the energy up-converted emission.

For the $2.34 \mathrm{eV}$ illumination and with the second photon within the range of ionization transitions of ZnSe acceptors, illumination quenches the DAP intensity (Fig. 8). Photo-ionization of shallow acceptors reduces also the population of the $\mathrm{Cr}^{1+}$ charge state. This is observed as a rapid photo-induced quenching of the $\mathrm{Cr}^{1+}$ signal in the photo-ESR study (see Fig. 2).

The up-conversion process is also deactivated when shallow acceptors are thermally ionized. Use of host material with larger acceptor ionization energies is thus profitable. In fact, we observed better temperature stability of energy up-converted DAP emission in $\mathrm{ZnCdS}: \mathrm{Cr}$ and $\mathrm{ZnSSe}: \mathrm{Cr}$ than in $\mathrm{ZnSe}: \mathrm{Cr}$. This is due to the fact that acceptors are deeper in these two alloys than those in $\mathrm{ZnSe}$ lattice.

\section{Infrared intra-shell emission of chromium 2+}

In Fig. 9 we show three PL emission efficiently excited in $\mathrm{ZnSe}$ : $\mathrm{Cr}$ under $\mathrm{Cr}$ photo-ionization transition. In addition to the blue DAP emission, two infrared emissions, with maximum at about $0.95 \mu \mathrm{m}$ and $2.4 \mu \mathrm{m}$ are observed. Both these infrared bands are relatively broad, which can result in optically pumped and tunable laser emission with the wavelength of emission being suitable for some optoelectronic applications, such as surgery lasers, for remote sensing, gas sensing, etc. [14-18].

We found that the all three PL emissions have the same excitation bands and that the two channels of recombination (visible versus infrared) compete. At low

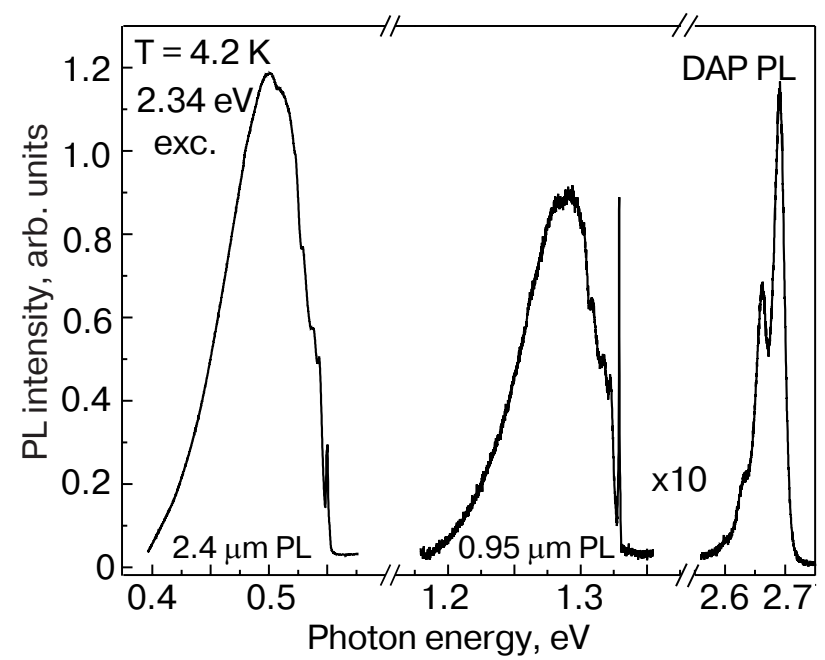

Fig. 9. Two infrared intra-shell emissions of chromium 2+ in $\mathrm{ZnSe}$ shown together with the blue energy up-converted DAP emission. temperatures the blue DAP emission dominates, but at increased temperatures the infrared emissions become more pronounced and dominate at room temperature.

The Cr photo-ionization band is characterized by large oscillator strength, i.e., the resulting photo-excitation can be more efficient in excitation of infrared emissions than the direct intra-shell excitation. Thus, such excitation can result in more efficient optical pumping of $\mathrm{Cr}^{2+}$ intra-shell emissions. This fact is important considering intensive studies on laser action on $\mathrm{Cr} 2+$ intra-shell transitions.

\section{Conclusions and Summary}

Initial interest in $\mathrm{ZnSe}$ started from observation of fairly efficient energy up-conversion. Blue PL emission could be observed under optical pumping with green light. Detail studies indicated that the process is efficient only at low temperatures. Also pumping energy was not optimized for excitation with red color laser diodes. Both temperature stability of the process and pumping energy can be improved/tuned if we use solid alloys of II-VI, such as ZnCdS or ZnSSe. Meanwhile it turned out that the system is suitable for tunable mid-infrared emission, using intra-shell transitions of chromium 2+. Mid-infrared emission with power above $1 \mathrm{~W}$ has already been achieved, making the system very attractive for practical applications.

This work was partly supported by the RFBR-Ural (Grant 04-02-96096).

1. V.Yu. Ivanov, Yu.G. Semenov, M. Surma, and M. Godlewski, Phys. Rev. B54, 4696 (1996).

2. M. Godlewski, A.J. Zakrzewski, and V.Y. Ivanov, J. Alloys Compounds 300/301, 23 (2000).

3. M. Godlewski and A. Zakrzewski, in: $I I-V I$ Semiconductors, M. Jain (ed.), World Scientific, Singapore (1993), p. 205.

4. M. Godlewski and M. Kaminska, J. Phys. C13, 6537 (1980).

5. M. Godlewski, Z. Wilamowski, M. Kaminska, W.E. Lamb, and B.C. Cavenett, J. Phys. C14, 2835 (1981).

6. M. Surma, M. Godlewski, and T.P. Surkova, Phys. Rev. B50, 8319 (1994).

7. M. Godlewski and A. Zakrzewski, J. Phys. C18, 6615 (1985).

8. M. Godlewski, Phys. Rev. B32, 8162 (1985).

9. A. Zakrzewski and M. Godlewski, Appl. Surf. Science 50, 257 (1991).

10. A. Zakrzewski and M. Godlewski, J. Appl. Phys. 67, 2457 (1990).

11. M. Surma and M. Godlewski, Acta Phys. Pol. A92, 1017 (1997).

12. V.Yu. Ivanov, G. Karczewski, M. Godlewski, A.R. Omel'chuk, A.E. Belyaev, and N.V. Zhavoronkov, Acta Phys. Pol. A103, 695 (2003). 
13. T.P. Surkova, M. Godlewski, K. Swiatek, P. Kaczor, A. Polimeni, L. Eaves, and W. Giriat, Physica B273-274, 848 (1999).

14. R.H. Page, K.I. Schaffers, L.D. DeLoach, G.D. Wilke, F.D. Patel, J.B. Tassano, Jr., S.A. Payne, W.F. Krupke, Kuo-Tong Chen, and A. Burger, IEEE J. Quantum Electron. 33, 609 (1997).

15. S. Bhaskar, P.S. Dobal, B.K. Rai, R.S. Katiyar, H.D. Bist, J.-O. Ndap, and A. Burger, J. Appl. Phys. 85, 439 (1999).
16. A. Burger, K. Chattopadhyay, J.-O. Ndap, X. Ma, S.H. Morgan, C.I. Rablau, C.-H. Su, S. Feth, R.H. Page, K.I. Schaffers, and A. Payne, J. Cryst. Growth 225, 249 (2001).

17. A.V. Podlipensky, V.G. Shcherbitsky, N.V. Kuleshov, V.I. Levchenko, V.N. Yakimovich, M. Mond, E. Heumann, G. Huber, H. Kretschmann, and S. Kuck, Appl. Phys. B72, 253 (2001).

18. E. Sorokin and I.T. Sorokina, Appl. Phys. Lett. 80, 3289 (2002). 